\title{
New Oscillation Results for Forced Second Order Differential Equations with Mixed Nonlinearities
}

\author{
Ercan Tunç, Adil Kaymaz \\ Department of Mathematics, Faculty of Arts and Sciences, Gaziosmanpaşa University, Tokat, Turkey \\ Email: ercantunc72@yahoo.com, adilkaymaz@gmail.com
}

Received November 17, 2011; revised December 17, 2011; accepted December 25, 2011

\begin{abstract}
Some new oscillation criteria are given for forced second order differential equations with mixed nonlinearities by using the generalized variational principle and Riccati technique. Our results generalize and extend some known oscillation results in the literature.
\end{abstract}

Keywords: Generalized Variational Principle; Variational Principle; Second Order Differential Equations; Riccati Transformation; Oscillation

\section{Introduction}

The oscillatory behavior of second order differential equations has a major role in the theory of differential equations. It has been shown that many real world problems can be modelled, in particular, by half linear differential equations which can be regarded as a natural generalization of linear differential equations [114]. A considerable amount of research has also been done on quasi-linear [15-18] and nonlinear second order differential equations [19-23].

In this paper, we investigate the oscillatory behavior of second order forced differential equation with mixed nonlinearities.

$$
\begin{aligned}
& \left(r(t)\left|x^{\prime}(t)\right|^{\alpha-1} x^{\prime}(t)\right)^{\prime}+p(t)|x(t)|^{\alpha-1} x(t) \\
& +\sum_{j=1}^{m} q_{j}(t)|x(t)|^{\beta_{j}-1} x(t)=e(t), t \geq t_{0},
\end{aligned}
$$

where $r \in C\left(\left[t_{0}, \infty\right),(0, \infty)\right)$, $p, q_{j}(1 \leq j \leq m), e \in C\left(\left[t_{0}, \infty\right),(-\infty, \infty)\right)$ and $0<\alpha<\beta_{1}<\beta_{2}<\cdots<\beta_{m}$ are real numbers, $p, q_{j} \quad(1 \leq j \leq m)$ and $e$ might alternate signs.

By a solution of Equation (1), we mean a function $x(t) \in C^{1}\left(\left[T_{x}, \infty\right),(-\infty, \infty)\right)$, where $T_{x} \geq t_{0}$ depends on the particular solution, which has the property that $r(t)\left|x^{\prime}(t)\right|^{\alpha-1} x^{\prime}(t) \in C^{1}\left[T_{x}, \infty\right)$ and satisfies Equation (1). We restrict our attention to the nontrivial solutions $x(t)$ of Equation (1) only, i.e., to solutions $x(t)$ such that $\sup \{|x(t)|: t \geq T\}>0$ for all $T \geq T_{x}$. A nontrivial solution of (1) is oscillatory if it has arbitrarily large zeros, otherwise, it is called non-oscillatory.
Equation (1) is said to be oscillatory if all its nontrivial solutions are oscillatory.

Equation (1) and its special cases such as the linear differential equation

$$
\left(r(t) x^{\prime}(t)\right)^{\prime}+q(t) x(t)=e(t),
$$

the half-linear differential equation

$$
\left(r(t)\left|x^{\prime}(t)\right|^{\alpha-1} x^{\prime}(t)\right)^{\prime}+q(t)|x(t)|^{\alpha-1} x(t)=e(t)
$$

and the quasi-linear differential equation

$$
\left(r(t)\left|x^{\prime}(t)\right|^{\alpha-1} x^{\prime}(t)\right)^{\prime}+q(t)|x(t)|^{\beta-1} x(t)=e(t)
$$

have been extensively studied by numerous authors with different methods (see, for example, [1-5,15-19] and the references quoted therein).

In 1999, Wong [1] proved the following theorem by making use of the "oscillatory intervals" of $e(t)$ and Leighton's variational principle (see [10]) for (2).

Theorem 1.1. Suppose that for any $T \geq t_{0}$, there exist $T \leq s_{1}<t_{1} \leq s_{2}<t_{2}$ such that

$$
e(t)\left\{\begin{array}{l}
\leq 0, t \in\left[s_{1}, t_{1}\right] \\
\geq 0, t \in\left[s_{2}, t_{2}\right] .
\end{array}\right.
$$

Denote

$D\left(s_{i}, t_{i}\right)=\left\{u \in C^{1}\left[s_{i}, t_{i}\right]: u(t) \not \equiv 0, u\left(s_{i}\right)=u\left(t_{i}\right)=0\right\}$, $i=1,2$.

If there exist $u \in D\left(s_{i}, t_{i}\right)$ such that 


$$
Q_{i}(u)=\int_{s_{i}}^{t_{i}}\left[q(t) u^{2}(t)-r(t)\left(u^{\prime}(t)\right)^{2}\right] \mathrm{d} t \geq 0, i=1,2
$$

then Equation (2) is oscillatory.

Afterwards, in 2002, the authors of [2] extended Wong's results, using a similar method, to Equation (3) as follows.

Theorem 1.2. Suppose that for any $T \geq t_{0}$, there exist $T \leq s_{1}<t_{1} \leq s_{2}<t_{2}$ such that (5) holds. Let

$$
\begin{aligned}
& D\left(s_{i}, t_{i}\right)=\left\{u \in C^{1}\left[s_{i}, t_{i}\right]: u(t) \not \equiv 0, u\left(s_{i}\right)=u\left(t_{i}\right)=0\right\}, \\
& i=1,2 .
\end{aligned}
$$

If there exist $H \in D\left(s_{i}, t_{i}\right)$ and a positive, nondecreasing function $\phi \in C^{1}\left(\left[t_{0}, \infty\right)\right)$ such that

$$
\begin{aligned}
& \int_{s_{i}}^{t_{i}} H^{2}(t) \phi(t) q(t) \mathrm{d} t \\
& >K \int_{s_{i}}^{t_{i}} \frac{r(t) \phi(t)}{|H(t)|^{\alpha-1}}\left(2\left|H^{\prime}(t)\right|+|H(t)| \frac{\phi^{\prime}(t)}{\phi(t)}\right)^{\alpha+1} \mathrm{~d} t,
\end{aligned}
$$

for $i=1,2$, where $K=(1 /(\alpha+1))^{\alpha+1}$, then (3) is oscillatory.

Later, in 2007, Zheng and Meng [16], considering a more general Equation (4), improved the paper [2] and showed that the results obtained in [2] for Equation (3) can not be applied to the case $\alpha>1$. The main result of Zheng and Meng [16] is the following.

Theorem 1.3. Assume that for any $T \geq t_{0}$, there exist $T \leq s_{1}<t_{1} \leq s_{2}<t_{2}$ such that (5) holds. Let

$$
D\left(s_{i}, t_{i}\right)=\left\{u \in C^{1}\left[s_{i}, t_{i}\right]: u^{\alpha+1}(t)>0, t \in\left(s_{i}, t_{i}\right) \text { and } u\left(s_{i}\right)=u\left(t_{i}\right)=0\right\} \text { for } i=1,2 .
$$

Suppose that there exist $H \in D\left(s_{i}, t_{i}\right)$ and a positive, nondecreasing function $\phi \in C^{1}\left(\left[t_{0}, \infty\right),(-\infty, \infty)\right)$ such that

$$
Q_{i}^{\phi}(H):=\int_{s_{i}}^{t_{i}} \phi(t)\left[Q(t) H^{\alpha+1}(t)-r(t)\left(\left|H^{\prime}(t)\right|+\frac{H(t) \phi^{\prime}(t)}{(\alpha+1) \phi(t)}\right)^{\alpha+1}\right] \mathrm{d} t>0
$$

for $i=1,2$. Then Equation (4) is oscillatory, where

$$
Q(t)=\alpha^{-\alpha / \beta} \beta((\beta-\alpha))^{(\alpha-\beta) / \beta}[q(t)]^{\alpha / \beta}|e(t)|^{(\beta-\alpha) / \beta}, 0<\alpha \leq \beta
$$

with the convention that $0^{0}=1$.

Also, in [2009], Zheng et al. [17] extended the results obtained for Equation (4) to Equation (1) as follows.
Theorem 1.4. Assume that for any $T \geq t_{0}$, there exist $T \leq s_{1}<t_{1} \leq s_{2}<t_{2}$ such that $q_{j}(t) \geq 0(1 \leq j \leq m)$ for $t \in\left[s_{1}, t_{1}\right] \cup\left[s_{2}, t_{2}\right]$ and (5) holds. Let

$$
D\left(s_{i}, t_{i}\right)=\left\{u \in C^{1}\left[s_{i}, t_{i}\right]: u^{\alpha+1}(t)>0, t \in\left(s_{i}, t_{i}\right) \text { and } u\left(s_{i}\right)=u\left(t_{i}\right)=0\right\} \text { for } i=1,2 .
$$

If there exist $H \in D\left(s_{i}, t_{i}\right)$ and a positive function $\phi \in C^{1}\left(\left[t_{0}, \infty\right), R\right)$ such that

$$
\int_{s_{i}}^{t_{i}} \phi(t)\left[\left(p(t)+\sum_{j=1}^{m} Q_{j}(t)\right) H^{\alpha+1}(t)-r(t)\left(\left|H^{\prime}(t)\right|+\frac{H(t) \phi^{\prime}(t)}{(\alpha+1) \phi(t)}\right)^{\alpha+1}\right] \mathrm{d} t>0
$$

for $i=1,2$. Then Equation (1) is oscillatory, where

$$
Q_{j}(t)=\alpha^{-\alpha / \beta_{j}} \beta_{j}\left[m\left(\beta_{j}-\alpha\right)\right]^{\left(\alpha-\beta_{j}\right) / \beta_{j}}\left[q_{j}(t)\right]^{\alpha / \beta_{j}}|e(t)|^{\left(\beta_{j}-\alpha\right) / \beta_{j}}, \quad 1 \leq j \leq m
$$

with the convention that $0^{0}=1$.

Recently, Shao [15] generalized the results of Zheng and Meng [16] by using the generalized variational principle due to Komkov [24] and gave the following result for Equation (4).

Theorem 1.5. Assume that, for any $T \geq t_{0}$, there exist $T \leq s_{1}<t_{1} \leq s_{2}<t_{2}$ such that (1.5) holds. Let $u \in C^{1}\left[s_{i}, t_{i}\right]$, and nonnegative functions $G_{1}, G_{2}$ satisfying $G_{i}\left(u\left(s_{i}\right)\right)=G_{i}\left(u\left(t_{i}\right)\right)_{\alpha+1}=0, \quad g_{i}(u)=G_{i}^{\prime}(u)$ are continuous and $\left(g_{i}(u(t))\right)^{\alpha+1} \leq(\alpha+1)^{\alpha+1} G_{i}^{\alpha}(u(t))$ for $t \in\left[s_{i}, t_{i}\right], i=1,2$. If there exists a positive function $\phi \in C^{1}\left(\left[t_{0}, \infty\right), R\right)$ such that

$$
\begin{aligned}
& Q_{i}^{\phi}(u) \\
& :=\int_{s_{1}}^{t_{1}} \phi\left[Q G_{i}(u)-r\left(\left|u^{\prime}\right|+\frac{G_{i}^{1 /(\alpha+1)}(u)\left|\phi^{\prime}\right|}{(\alpha+1) \phi}\right)^{\alpha+1}\right](t) \mathrm{d} t>0
\end{aligned}
$$

for $i=1,2$, then Equation (4) is oscillatory, where $Q(t)$ is the same as (9). 
Motivated by the above theorems we propose some new oscillation results by employing the generalized variational principle and Riccati technique for Equation (1). Our results extend and generalize some known results in the literature. We now state our main results and several remarks.

\section{New Oscillation Results}

In order to prove our results we use the following wellknown inequality which is presented by Hardy et al. [25].

Lemma 2.1. (see [25]). If $X$ and $Y$ are nonnegative, then

$$
\begin{aligned}
X^{\gamma}+(\gamma-1) Y^{\gamma} & \geq \gamma X Y^{\gamma-1}, \gamma>1, \\
\int_{s_{i}}^{t_{i}} \phi(t) & {\left[\left(p(t)+\sum_{j=1}^{m} Q_{j}(t)\right) G_{i}(u(t))-r(t)\left(\left|u^{\prime}(t)\right|+\frac{G_{i}^{1 /(\alpha+1)}(u(t))\left|\phi^{\prime}(t)\right|}{(\alpha+1) \phi(t)}\right)^{\alpha+1}\right] \mathrm{d} t>0 }
\end{aligned}
$$

for $i=1,2$, then Equation (1) is oscillatory, where $Q_{j}(t)$ is the same as (11).

Proof. Suppose that $x=x(t)$ is a nonoscillatory solution of Equation (1). Then, there exists a $T_{0} \geq t_{0}$ such that $x(t) \neq 0$ for all $t \geq T_{0}$. Without loss of generality, we may assume that $x(t)>0$ for all where equality holds if and only if $X=Y$.

Theorem 2.1. Assume that, for any $T \geq t_{0}$, there exist $T \leq s_{1}<t_{1} \leq s_{2}<t_{2}$ such that $q_{j}(t) \geq 0(1 \leq j \leq m)$ for $t \in\left[s_{1}, t_{1}\right] \cup\left[s_{2}, t_{2}\right]$ and (5) holds. Let $u \in C^{1}\left[s_{i}, t_{i}\right]$ and nonnegative functions $G_{1}, G_{2}$ satisfying

$$
G_{i}\left(u\left(s_{i}\right)\right)=G_{i}\left(u\left(t_{i}\right)\right)=0, g_{i}(u)=G_{i}^{\prime}(u)
$$

are continuous and

$$
\left(g_{i}(u(t))\right)^{\alpha+1} \leq(\alpha+1)^{\alpha+1} G_{i}^{\alpha}(u(t))
$$

for $t \in\left[s_{i}, t_{i}\right], i=1,2$. If there exists a positive function $\phi \in C^{1}\left(\left[t_{0}, \infty\right), R\right)$ such that

$$
\begin{aligned}
w^{\prime}(t)= & \frac{\phi^{\prime}(t)}{\phi(t)} w(t) \\
& +\phi(t)\left[\frac{\left(-p(t)|x(t)|^{\alpha-1} x(t)-\sum_{j=1}^{m} q_{j}(t)|x(t)|^{\beta_{j}-1} x(t)+e(t)|x(t)|^{\alpha-1} x(t)-r(t)\left|x^{\prime}(t)\right|^{\alpha-1} x^{\prime}(t)\left(|x(t)|^{\alpha-1} x(t)\right)^{\prime}\right]}{\left(|x(t)|^{\alpha-1} x(t)\right)^{2}}\right] \\
= & \frac{\phi^{\prime}(t)}{\phi(t)} w(t)-\phi(t) p(t)-\phi(t)\left[\sum_{j=1}^{m} q_{j}(t)|x(t)|^{\beta_{j}-\alpha}-\frac{e(t)}{|x(t)|^{\alpha-1} x(t)}\right]-\alpha \frac{|w(t)|^{(\alpha+1) / \alpha}}{(\phi(t) r(t))^{1 / \alpha}} .
\end{aligned}
$$

By the assumption, we can choose $s_{1}, t_{1} \geq T_{0}$ so that $e(t) \leq 0$ on the interval $I_{1}=\left[s_{1}, t_{1}\right]$ with $s_{1}<t_{1}$. As in [18], for given $t \in I_{1}$, set

$$
F_{j}(s)=q_{j}(t) s^{\beta_{j}-\alpha}-\frac{e(t)}{m s^{\alpha}}, 1 \leq j \leq m, s>0 .
$$

It is easy to verify that

$$
\begin{aligned}
& F_{j}^{\prime}\left(s_{j}^{*}\right)=0 \text { and } F_{j}^{\prime \prime}\left(s_{j}^{*}\right)>0, \\
& \text { where } s_{j}^{*}=\left[\frac{-\alpha e(t)}{m\left(\beta_{j}-\alpha\right) q_{j}(t)}\right]^{1 / \beta_{j}} .
\end{aligned}
$$

Differentiating (15) and using (1), we obtain, for all $t \geq T_{0}$,

$$
w(t)=\phi(t) \frac{r(t)\left|x^{\prime}(t)\right|^{\alpha-1} x^{\prime}(t)}{|x(t)|^{\alpha-1} x(t)}, t \geq T_{0} .
$$




$$
\phi(t)\left(p(t)+\sum_{j=1}^{m} Q_{j}(t)\right) \leq-w^{\prime}(t)+\frac{\phi^{\prime}(t)}{\phi(t)} w(t)-\alpha \frac{|w(t)|^{(\alpha+1) / \alpha}}{(\phi(t) r(t))^{1 / \alpha}} .
$$

Multiplying $G_{1}(u(t))$ through (18) and integrating over $I_{1}$, we have

$$
\int_{s_{1}}^{t_{1}} \phi(t)\left(p(t)+\sum_{j=1}^{m} Q_{j}(t)\right) G_{1}(u(t)) \mathrm{d} t \leq-\int_{s_{1}}^{t_{1}} G_{1}(u(t)) w^{\prime}(t) \mathrm{d} t+\int_{s_{1}}^{t_{1}} G_{1}(u(t)) \frac{\phi^{\prime}(t)}{\phi(t)} w(t) \mathrm{d} t-\alpha \int_{s_{1}}^{t_{1}} G_{1}(u(t)) \frac{|w(t)|^{(\alpha+1) / \alpha}}{(\phi(t) r(t))^{1 / \alpha}} \mathrm{d} t .(19)
$$

By integration by parts and using the fact that $G_{1}\left(u\left(s_{1}\right)\right)=G_{1}\left(u\left(t_{1}\right)\right)=0$, we have

$$
\int_{s_{1}}^{t_{1}} G_{1}(u(t)) w^{\prime}(t) \mathrm{d} t=\left.G_{1}(u(t)) w(t)\right|_{s_{1}} ^{t_{1}}-\int_{s_{1}}^{t_{1}} G_{1}^{\prime}(u(t)) u^{\prime}(t) w(t) \mathrm{d} t=-\int_{s_{1}}^{t_{1}} g_{1}(u(t)) u^{\prime}(t) w(t) \mathrm{d} t .
$$

In view of (19) and (20), we conclude that

$$
\begin{aligned}
& \int_{s_{1}}^{t_{1}} \phi(t)\left(p(t)+\sum_{j=1}^{m} Q_{j}(t)\right) G_{1}(u(t)) \mathrm{d} t \\
& \leq \int_{s_{1}}^{t_{1}}\left[g_{1}(u(t)) u^{\prime}(t)+G_{1}(u(t)) \frac{\phi^{\prime}(t)}{\phi(t)}\right] w(t) \mathrm{d} t-\alpha \int_{s_{1}}^{t_{1}} G_{1}(u(t)) \frac{|w(t)|^{(\alpha+1) / \alpha}}{(\phi(t) r(t))^{1 / \alpha}} \mathrm{d} t \\
& \leq \int_{s_{1}}^{t_{1}}\left[\left|g_{1}(u(t))\right|\left|u^{\prime}(t)\right|+G_{1}(u(t)) \frac{\left|\phi^{\prime}(t)\right|}{\phi(t)}\right]|w(t)| d t-\alpha \int_{s_{1}}^{t_{1}} G_{1}(u(t)) \frac{|w(t)|^{(\alpha+1) / \alpha}}{(\phi(t) r(t))^{1 / \alpha}} \mathrm{d} t \\
& \leq(\alpha+1) \int_{s_{1}}^{t_{1}}\left[G_{1}^{\alpha /(\alpha+1)}(u(t))\left|u^{\prime}(t)\right|+G_{1}(u(t)) \frac{\left|\phi^{\prime}(t)\right|}{(\alpha+1) \phi(t)}\right]|w(t)| \mathrm{d} t-\alpha \int_{s_{1}}^{t_{1}} G_{1}(u(t)) \frac{|w(t)|^{(\alpha+1) / \alpha}}{(\phi(t) r(t))^{1 / \alpha}} \mathrm{d} t .
\end{aligned}
$$

Let

$$
X=\left[\frac{\alpha}{(\phi(t) r(t))^{1 / \alpha}}\right]^{\alpha /(\alpha+1)} G_{1}^{\alpha /(\alpha+1)}(u(t))|w(t)|, \quad \gamma=\frac{\alpha+1}{\alpha}, Y=(\alpha \phi(t) r(t))^{\alpha /(\alpha+1)}\left[\left|u^{\prime}(t)\right|+\frac{G_{1}^{1 /(\alpha+1)}(u(t))\left|\phi^{\prime}(t)\right|}{(\alpha+1) \phi(t)}\right]^{\alpha} .
$$

According to Lemma 2.1, we obtain for $t \in\left[s_{1}, t_{1}\right]$

$$
\begin{aligned}
& (\alpha+1)\left[G_{1}^{\alpha /(\alpha+1)}(u(t))\left|u^{\prime}(t)\right|+G_{1}(u(t)) \frac{\left|\phi^{\prime}(t)\right|}{(\alpha+1) \phi(t)}\right]|w(t)|-\alpha G_{1}(u(t)) \frac{|w(t)|^{(\alpha+1) / \alpha}}{(\phi(t) r(t))^{1 / \alpha}} \\
& \leq \frac{1}{\alpha}(\alpha \phi(t) r(t))\left[\left|u^{\prime}(t)\right|+\frac{G_{1}^{1 /(\alpha+1)}(u(t))\left|\phi^{\prime}(t)\right|}{(\alpha+1) \phi(t)}\right]^{\alpha+1}=\phi(t) r(t)\left[\left|u^{\prime}(t)\right|+\frac{G_{1}^{1 /(\alpha+1)}(u(t))\left|\phi^{\prime}(t)\right|}{(\alpha+1) \phi(t)}\right]^{\alpha+1} .
\end{aligned}
$$

Therefore, (21) yields

$$
\int_{s_{1}}^{t_{1}} \phi(t)\left(p(t)+\sum_{j=1}^{m} Q_{j}(t)\right) G_{1}(u(t)) \mathrm{d} t \leq \int_{s_{1}}^{t_{1}} \phi(t) r(t)\left[\left|u^{\prime}(t)\right|+\frac{G_{1}^{1 /(\alpha+1)}(u(t))\left|\phi^{\prime}(t)\right|}{(\alpha+1) \phi(t)}\right]^{\alpha+1} \mathrm{~d} t,
$$

which contradicts the assumption (14) for $i=1$.

When $x(t)$ is a negative solution for $t \geq T_{0}>t_{0}$, we may employ the fact that $e(t) \geq 0$ on $I_{2}=\left[s_{2}, t_{2}\right]$ to reach a similar contradiction. Therefore, any solution $x(t)$ can be neither eventually positive nor eventually negative. Hence, any solution is oscillatory. This completes the proof of Theorem 2.1.

If $p(t) \equiv 0$ and $m=1$, then Equation (1) reduces to Equation (4). Thus by Theorem 2.1, we have the following oscillation result: 
Corollary 2.1. Assume that, for any $T \geq t_{0}$, there exist $T \leq s_{1}<t_{1} \leq s_{2}<t_{2}$ such that (5) holds. Let $u \in C^{1}\left[s_{i}, t_{i}\right]$, and nonnegative functions $G_{1}, G_{2}$ satisfying $G_{i}\left(u\left(s_{i}\right)\right)=G_{i}\left(u\left(t_{i}\right)\right)=0, \quad g_{i}(u)=G_{i}^{\prime}(u)$ are continuous and $\left(g_{i}(u(t))\right)^{\alpha+1} \leq(\alpha+1)^{\alpha+1} G_{i}^{\alpha}(u(t))$ for $t \in\left[s_{i}, t_{i}\right]$ for $i=1,2$. If there exists a positive function $\phi \in C^{1}\left(\left[t_{0}, \infty\right), R\right)$ such that

$$
\int_{s_{i}}^{t_{i}} \phi(t)\left[Q(t) G_{i}(u(t))-r(t)\left(\left|u^{\prime}(t)\right|+\frac{G_{i}^{1 /(\alpha+1)}(u(t))\left|\phi^{\prime}(t)\right|}{(\alpha+1) \phi(t)}\right)^{\alpha+1}\right] \mathrm{d} t>0
$$

for $i=1,2$, then Equation (4) is oscillatory, where $Q(t)$ is the same as (9).

Remark 1. Corollary 2.1 shows that Theorem 2.1 is a generalization of Theorem 1.5.

Remark 2. Let $G_{1}(u)=G_{2}(u)=u^{\alpha+1}$ in Corollary 2.1, then our main Theorem 2.1 reduces to Theorem 1.3 .

Remark 3. If we choose $G_{1}(u)=G_{2}(u)=u^{\alpha+1}$ in Theorem 2.1, then we obtain Theorem 1.4.

Remark 4. If we choose $G_{1}(u)=G_{2}(u)=u^{\alpha+1}$ and $\phi(t) \equiv 1$ in Theorem 2.1, then we obtain Corollary 2.3 of Paper [17].

Remark 5. If we choose $G_{1}(u)=G_{2}(u)=u^{\alpha+1}$ and $\phi(t) \equiv 1$ in Corollary 2.1, then we obtain Corollary 2.3 of paper [16].

Remark 6. Let

$$
\begin{gathered}
G_{1}(u)=G_{2}(u)=u^{\alpha+1}, q_{j}(t) \equiv 0,1 \leq j \leq m \\
\left(\gamma t^{\lambda / 3}\left|x^{\prime}(t)\right|^{\alpha-1} x^{\prime}(t)\right)^{\prime}+t^{\lambda / 3}|x(t)|^{\alpha-1} x(t)+t^{\lambda}|x(t)|^{\beta-1} x(t)=-\sin ^{3} t,
\end{gathered}
$$

for $t \geq 1$, where $\gamma, \lambda>0$ are constants. Let $\alpha=1$ and $\beta=3$, so $Q(t)=(3 / \sqrt[3]{4}) t^{\lambda / 3} \sin ^{2} t$. The zeros of forcing term $-\sin ^{3} t$ are $n \pi$. For any $T \geq 1$, we choose $n$ sufficiently large so that $n \pi=2 k \pi \geq T$, and $\phi(t) \equiv 1$ in Theorem 2.1, then Theorem 2.1 is a generalization of Theorem 1.1.

Remark 7. Let $q_{j}(t) \equiv 0,(1 \leq j \leq m)$. If we choose $G_{1}(u)=G_{2}(u)=u^{\alpha+1}$ in Theorem 2.1, then Theorem 2.1 improves Theorem 1.2, since the positive constant $\alpha$ in Theorem 2.1 can be chosen as any number lying in $(0, \infty)$.

Remark 8. If the condition (5) in Theorem 2.1 and Corollary 2.1 is replaced by

$$
e(t)\left\{\begin{array}{l}
\geq 0, t \in\left[s_{1}, t_{1}\right] \\
\leq 0, t \in\left[s_{2}, t_{2}\right]
\end{array}\right.
$$

then the results given in this paper are still valid.

\section{Examples}

$$
\int_{s_{1}}^{t_{1}} \phi(t)(p(t)+Q(t)) G_{i}(u(t)) \mathrm{d} t=\int_{2 k \pi}^{(2 k+1) \pi}\left(\sin ^{2} t+(3 / \sqrt[3]{4}) \sin ^{4} t\right) \exp (-\sin t) \mathrm{d} t \geq \frac{\pi}{2 e}\left(1+\frac{9}{4 \sqrt[3]{4}}\right),
$$

and

$$
\int_{s_{1}}^{t_{1}} \phi(t) r(t)\left(\left|u^{\prime}(t)\right|+\frac{G_{i}^{1 /(\alpha+1)}(u(t))\left|\phi^{\prime}(t)\right|}{(\alpha+1) \phi(t)}\right)^{\alpha+1} \mathrm{~d} t=\int_{2 k \pi}^{(2 k+1) \pi} \gamma\left(|\cos t|+\frac{\lambda \sin t \exp (-\sin t / 2)}{6 t}\right)^{2} \mathrm{~d} t<\gamma\left(1+\frac{\lambda}{6}\right)^{2} \pi
$$

Therefore, Equation (14) is satisfied for $i=1$ provided that $0<\gamma<\frac{1}{2 e}\left(1+\frac{9}{4 \sqrt[3]{4}}\right) /\left(1+\frac{\lambda}{6}\right)^{2}$. In a similar way, for $s_{2}=(2 k+1) \pi$ and $t_{2}=(2 k+2) \pi$, we choose $u(t)=\sin t \leq 0, G_{2}(u)=u^{2} \exp (u)$ (it is easy to verify that $\left(G_{2}{ }^{\prime}(u)\right)^{2} \leq 4 G_{2}(u)$ for $\left.u \leq 0\right)$ so that $s_{1}=2 k \pi$ and $t_{1}=(2 k+1) \pi$. Letting

$u(t)=\sin t \geq 0, \quad G_{1}(u)=u^{2} \exp (-u) \quad$ (it is easy to verify that $\left(G_{1}^{\prime}(u)\right)^{2} \leq 4 G_{1}(u)$ for $\left.u \geq 0\right), \phi(t)=t^{-\lambda / 3}$, then we obtain that (14) is valid for $i=2$. Thus (23) is oscillatory for

$$
0<\gamma<\frac{1}{2 e}\left(1+\frac{9}{4 \sqrt[3]{4}}\right) /\left(1+\frac{\lambda}{6}\right)^{2}
$$

by Theorem 2.1 .

Example 3.2. Consider the following forced quasilinear differential equation 


$$
\left[\gamma(2+\cos t) t^{-\lambda / 5}\left|x^{\prime}\right|^{\alpha-1} x^{\prime}\right]^{\prime}+t^{-\lambda / 5} \exp (\sin t / 5)|x|^{\alpha-1} x+t^{-\lambda} \exp (\sin t)|x|^{\beta-1} x=-\sin ^{5} t
$$

for $t \geq 1$, where $\gamma, \lambda>0$ are constants. Let $\alpha=1$ and $\beta=5$, so $Q(t)=\frac{5}{\sqrt[5]{4^{4}}} t^{-\lambda / 5} \exp (\sin t / 5) \sin ^{4} t$. The zeros of forcing term $-\sin ^{5} t$ are $n \pi$. For any $T \geq 1$, we choose $n$ sufficiently large so that $n \pi=2 k \pi \geq T, s_{1}=2 k \pi$ and $t_{1}=(2 k+1) \pi$. Letting $u(t)=\sin t \geq 0, G_{1}(u)=u^{2} \exp (-u), \phi(t)=t^{\lambda / 5}$,

then we obtain

$$
\begin{aligned}
\int_{s_{1}}^{t_{1}} \phi(t)(p(t)+Q(t)) G_{i}(u(t)) \mathrm{d} t & =\int_{2 k \pi}^{(2 k+1) \pi}\left(\sin ^{2} t+\frac{5}{\sqrt[5]{4^{4}}} \sin ^{6} t\right) \exp \left(\frac{-4 \sin t}{5}\right) \mathrm{d} t \\
& \geq \frac{1}{e^{4 / 5}} \int_{0}^{\pi}\left(\sin ^{2} t+\frac{5}{\sqrt[5]{4^{4}}} \sin ^{6} t\right)=\frac{\pi}{16 e^{4 / 5}}\left(8+\frac{25}{\sqrt[5]{4^{4}}}\right)
\end{aligned}
$$

and

$$
\begin{aligned}
\int_{s_{1}}^{t_{1}} \phi(t) r(t)\left(\left|u^{\prime}(t)\right|+\frac{G_{i}^{1 /(\alpha+1)}(u(t))\left|\phi^{\prime}(t)\right|}{(\alpha+1) \phi(t)}\right)^{\alpha+1} \mathrm{~d} t & =\int_{2 k \pi}^{(2 k+1) \pi} \gamma(2+\cos t)\left(|\cos t|+\frac{\lambda \sin t \exp (-\sin t / 2)}{10 t}\right)^{2} \mathrm{~d} t \\
& <\int_{0}^{\pi} 3 \gamma(1+\lambda / 10)^{2} \mathrm{~d} t=3 \gamma(1+\lambda / 10)^{2} \pi
\end{aligned}
$$

Therefore, Equation (14) is satisfied for $i=1$ provided that $0<\gamma<\frac{\delta}{3(1+\lambda / 10)^{2}}$, where $\delta=\frac{1}{16 e^{4 / 5}}\left(8+\frac{25}{\sqrt[5]{4^{4}}}\right)$.

In a similar way, for $s_{2}=(2 k+1) \pi$ and $t_{2}=(2 k+2) \pi$, we choose $u(t)=\sin t \leq 0$, $G_{2}(u)=u^{2} \exp (u)$ so that (14) is valid for $i=2$. Thus (24) is oscillatory for $0<\gamma<\delta / 3(1+\lambda / 10)^{2}$ by Theorem 2.1 .

\section{Conclusion}

The oscillatory behavior of many different kinds of differential equations has been investigated and a great deal of results has been obtained in the literature. In this article, we generalized the results obtained in [16,17] and extended the results of Shao [15] by using the generalized variational principle and Riccati tecnique. In a similar way, the results obtained for Equation (1) can be extended to a more general class of differential equations.

\section{Acknowledgements}

The authors would like to express sincere thanks to the anonymous referee for her/his invauable corrections, comments and suggestions on the paper.

\section{REFERENCES}

[1] J. S. W. Wong, "Oscillation Criteria for a Forced SecondOrder Linear Differential Equation," Journal of Mathe- matical Analysis and Applications, Vol. 231, No. 1, 1999, pp. 235-240. doi:10.1006/jmaa.1998.6259

[2] W. T. Li and S. S. Cheng, "An Oscillation Criterion for Nonhomogeneous Half-Linear Differential Equations," Applied Mathematics Letters, Vol. 15, No. 3, 2002, pp. 259-263. doi:10.1016/S0893-9659(01)00127-6

[3] J. V. Manojlovic, "Oscillation Criteria for Second-Order Half-Linear Differential Equations," Mathematical and Computer Modelling, Vol. 30, No. 5-6, 1999, pp. 109119. doi:10.1016/S0895-7177(99)00151-X

[4] Q. R. Wang, "Oscillation and Asymptotics for SecondOrder Half-Linear Differential Equations," Applied Mathematics and Computation, Vol. 122, No. 2, 2001, pp. 253266. doi:10.1016/S0096-3003(00)00056-4

[5] Q. R. Wang and Q. G. Yang "Interval Criteria for Oscillation of Second-Order Half-Linear Differential Equations," Journal of Mathematical Analysis and Applications, Vol. 291, No. 1, 2004, pp. 224-236. doi:10.1016/j.jmaa.2003.10.028

[6] J. Jaros and T. Kusano, "A Picone Type Identity for Second Order Half-Linear Differential Equations," Acta Mathematica Universitatis Comenianae, Vol. 68, No. 1, 1999, pp. 137-151.

[7] A. Elbert, "A Half-Linear Second Order Differential Equation," Colloquia Mathematica Societatis Janos Bolyai: Qualitative Theory of Differential Equations, Szeged, 1979, pp. 153-180.

[8] A. Wintner, "A Criterion of Oscillatory Stability," Quarterly of Applied Mathematics, Vol. 7, 1949, pp. 115-117.

[9] I. V. Kamenev, "An Integral Criterion for Oscillation of Linear Differential Equations of Second Order," Matematicheskie Zametki Vol. 23, No. 2, 1978, pp. 249-251. 
[10] W. Leighton, "Comparison Theorems for Linear Differential Equations of Second Order," Proceedings of the American Mathematical Society, Vol. 13, 1962, pp. 603610. doi:10.1090/S0002-9939-1962-0140759-0

[11] Q. Kong, "Interval Criteria for Oscillation of SecondOrder Linear Ordinary Differential Equation," Journal of Mathematical Analysis and Applications, Vol. 229, No. 1, 1999, pp. 258-270. doi:10.1006/jmaa.1998.6159

[12] H. J. Li and C. C. Yeh, "Sturm Comparison Theorem for Half-Linear Second Order Differential Equations," Proceedings of the Royal Society of Edinburgh, Vol. A125, 1995, pp. 1193-1240. doi:10.1017/S0308210500030468

[13] O. Došlý and P. Řehák, "Half-Linear Differential Equations," North-Holland Mathematics Studies, Vol. 202, Elsevier Science, Amsterdam, 2005.

[14] R. P. Agarwal, S. R. Grace and D. O'Regan, "Oscillation Theory for Second Order Linear, Half-Linear, Superlinear Dynamic Equations," Kluver, Dordrecht, 2002.

[15] J. Shao, "A New Oscillation Criterion for Forced SecondOrder Quasi-Linear Differential Equations," Discrete Dynamics in Nature and Society, Vol. 2011, Hindawi Publishing Corporation, New York, pp. 1-8.

[16] Z. Zheng and F. Meng, "Oscillation Criteria for Forced Second Order Quasi-Linear Differential Equations," Mathematical and Computer Modelling, Vol. 45, No. 1-2, 2007, pp. 215-220. doi:10.1016/j.mcm.2006.05.005

[17] Z. Zheng, X. Wang and H. Han, "Oscillation Criteria for Forced Second Order Differential Equations with Mixed Nonlinearities," Applied Mathematics Letters, Vol. 22, No. 7, 2009, pp. 1096-1101. doi:10.1016/j.aml.2009.01.018
[18] J. Jaros, T. Kusano and N. Yoshida, "Generalized Picone's Formula and Forced Oscillation in Quasi-Linear Differential Equations of the Second Order," Archivum Mathematicum, Vol. 38, No. 1, 2002, pp. 53-59.

[19] J. Shao and F. Meng, "Generalized Variational Principles on Oscillation for Nonlinear Nonhomogeneous Differential Equations," Abstract and Applied Analysis, Vol. 2011, 2011, pp. 1-10.

[20] Q. Yang, "Interval Oscillation Criteria for a Forced Second Order Nonlinear Ordinary Differential Equations with Oscillatory Potential," Applied Mathematics and Computation, Vol. 135, No. 1, 2003, pp. 49-64.

[21] D. Çakmak and A. Tiryaki, "Oscillation Criteria for Certain Forced Second-Order Nonlinear Differential Equations," Applied Mathematics Letters, Vol. 17, No. 3, 2004, pp. 275-279. doi:10.1016/S0893-9659(04)90063-8

[22] E. Tunç, "A Note on the Oscillation of Second-Order Differential Equations with Damping," Journal of Computational Analysis and Applications, Vol. 12, No. 2, 2010, pp. 444-453.

[23] E. Tunç, "Interval Oscillation Criteria for Certain Forced Second-Order Differential Equations," Carpathian Journal of Mathematics, Vol. 28, No. 1, 2012, in Press.

[24] V. Komkov, "A Generalization of Leighton's Variational Theorem," Applicable Analysis: An International Journal, Vol. 2, No. 4, 1972, pp. 377-383. doi:10.1080/00036817208839051

[25] G. H. Hardy, J. E. Littlewood and G. Polya, "Inequalities," 2nd Edition, Cambridge University Press, Cambridge, 1988. 